Supporting information

\title{
Preparation of Dual-template Epitope Imprinted Polymers for Targeted Fluorescence Imaging and Targeted Drug Delivery to Pancreatic Cancer BxPC-3 Cell
}

Chao Jia, ${ }^{\dagger}$ Man Zhang, ${ }^{\ddagger}$ Yan Zhang,,$^{\S}$ Zi-Bo Ma, ${ }^{\dagger}$ Nan-Nan Xiao, ${ }^{\S}$ Xi-Wen He, ${ }^{\dagger}$ Wen-You Li,

*,;, II Yu-Kui Zhang ${ }^{*}, \dagger, \perp$

$\dagger$ College of Chemistry, Research Center for Analytical Sciences, State Key Laboratory of

Medicinal Chemical Biology, Tianjin Key Laboratory of Biosensing and Molecular Recognition,

Nankai University, Tianjin 300071, China.

$\ddagger$ College of Pharmacy, Nankai University, Tianjin 300071, China.

$\S$ State Key Laboratory of Medicinal Chemical Biology, Nankai University, Tianjin 300350,

China.

॥Collaborative Innovation Center of Chemical Science and Engineering (Tianjin), Tianjin 300071, China.

$\perp$ National Chromatographic Research and Analysis Center, Dalian Institute of Chemical Physics, Chinese Academy of Sciences, Dalian 116023, China.

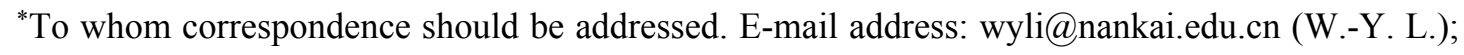
ykzhang@dicp.ac.cn (Y.-K. Z.). 

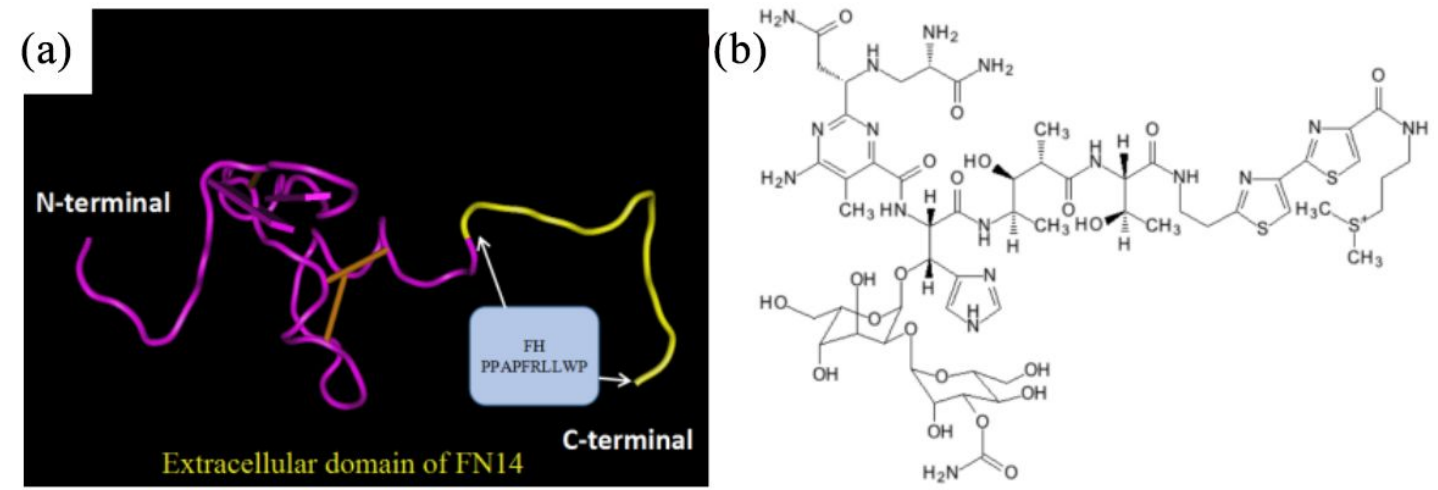

(c)

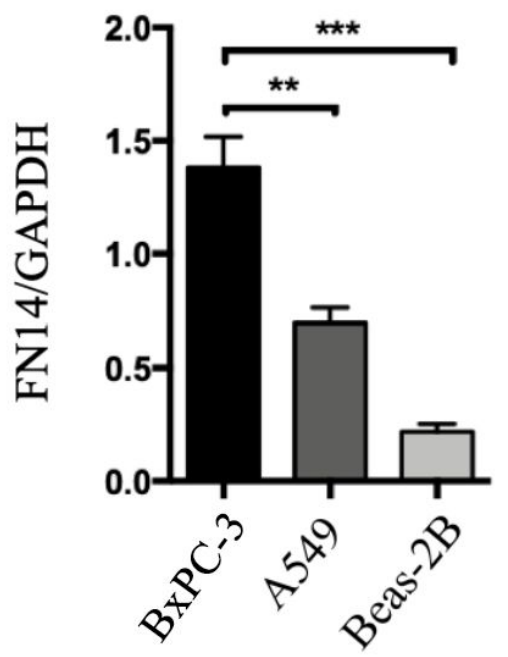

Figure S1. (a) Position of FH in extracellular domain of FN14; (b) Chemical structure of BLM; (c) The expression of FN14 on BxPC-3, A549 and Beas-2B cells. The relative intensity data of FN14 to GAPDH are presented as the mean $\pm \mathrm{SD}$ of three groups. $* * \mathrm{P}<0.01, * * * \mathrm{P}<0.001$ compared with the BxPC-3 group. 

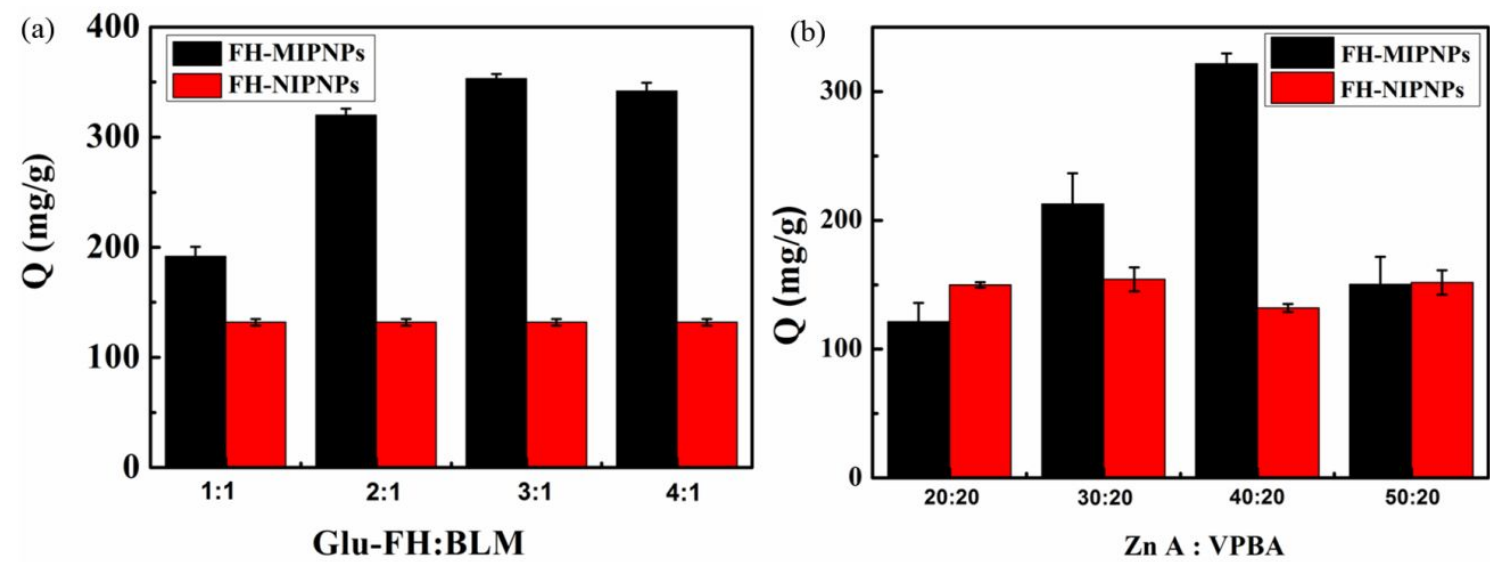

Figure S2. Optimization of adsorption effect by template dosage ratio (a) and function monomer dosage (b) during preparation. FH-MIPNPs or FH-NIPNPs $(0.5 \mathrm{mg} / \mathrm{ml})$ adsorbed to FH $(0.6$ $\mathrm{mg} / \mathrm{ml}$ ) under conditions of $\mathrm{pH}$ 7.4. The data show mean \pm s.d., representative from 3 independent experiments. 


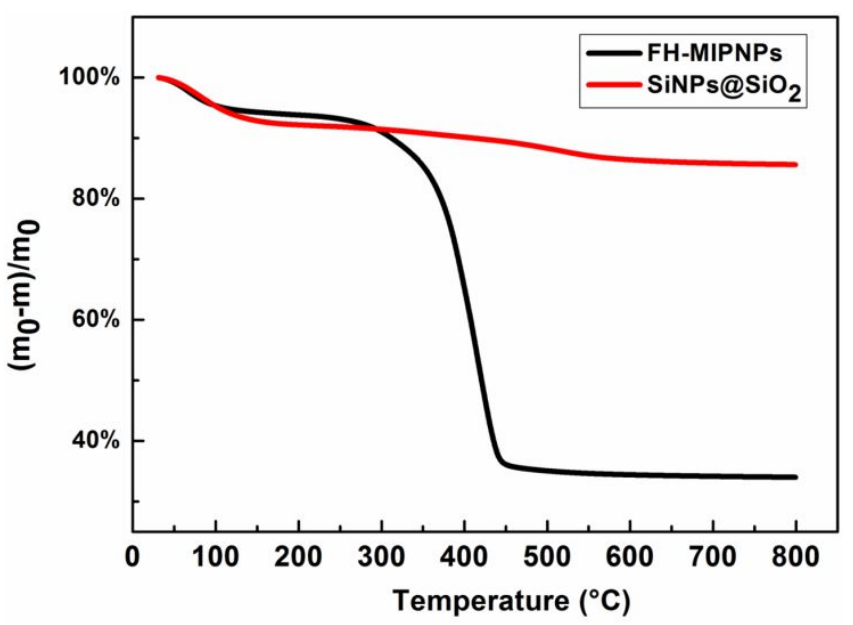

Figure S3. TGA curve of SiNPs@ $\mathrm{SiO}_{2}$ and FH-MIPNPs. 

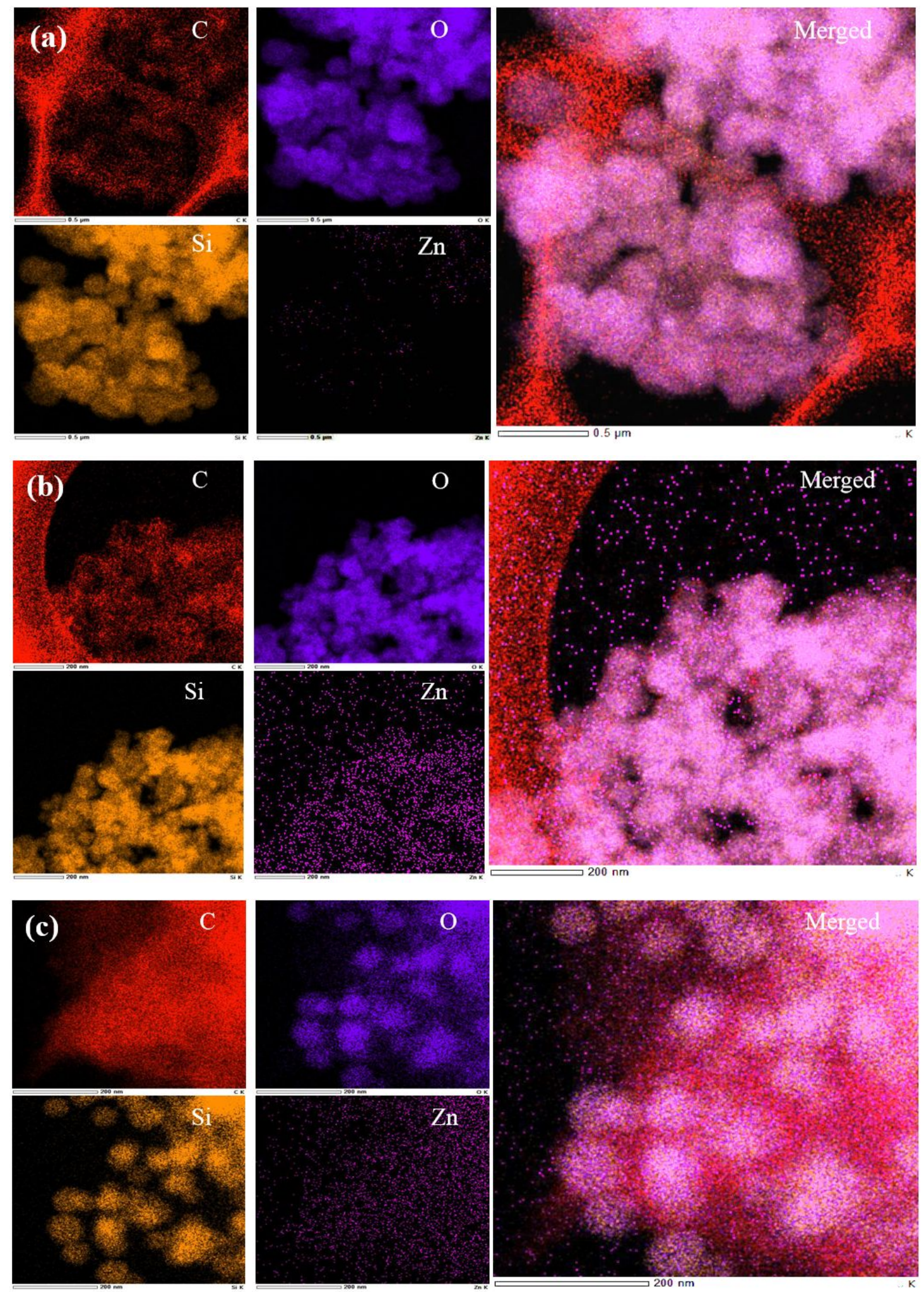

Figure S4. EDS elemental mapping of C, O, Si and Zn of (a) $\mathrm{SiNPs@SiO} 2$, (b) FH-NIPNPs and (c) FH-MIPNPs. 

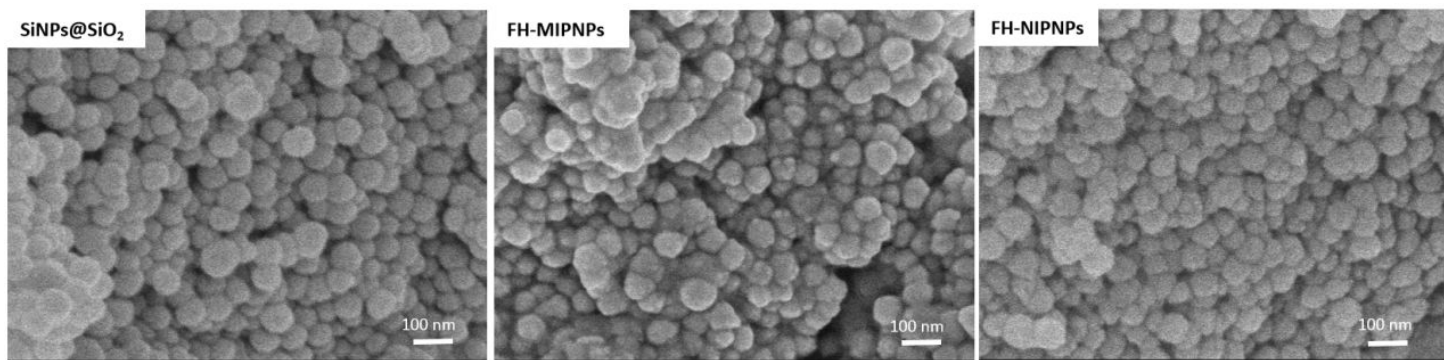

Figure S5. SEM imagine of SiNPs@ $\mathrm{SiO}_{2}$, FH-MIPNPs and FH-NIPNPs. 

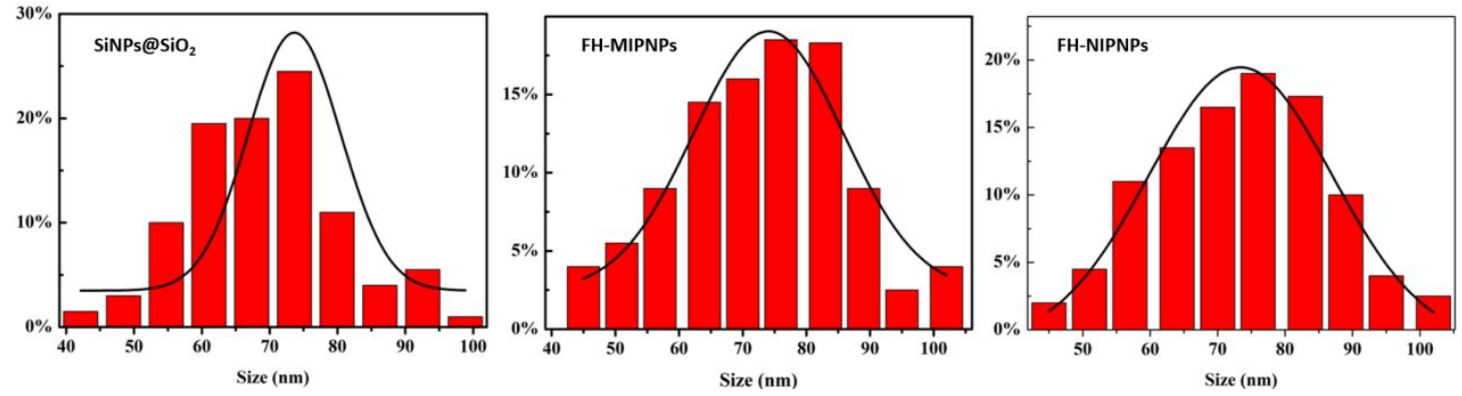

Figure S6. The particle size distribution statistics of SiNPs@SiO 2 , FH-MIPNPs and FH-NIPNPs, measured from SEM. More than 100 particles were counted for SEM analyses. 
(a)

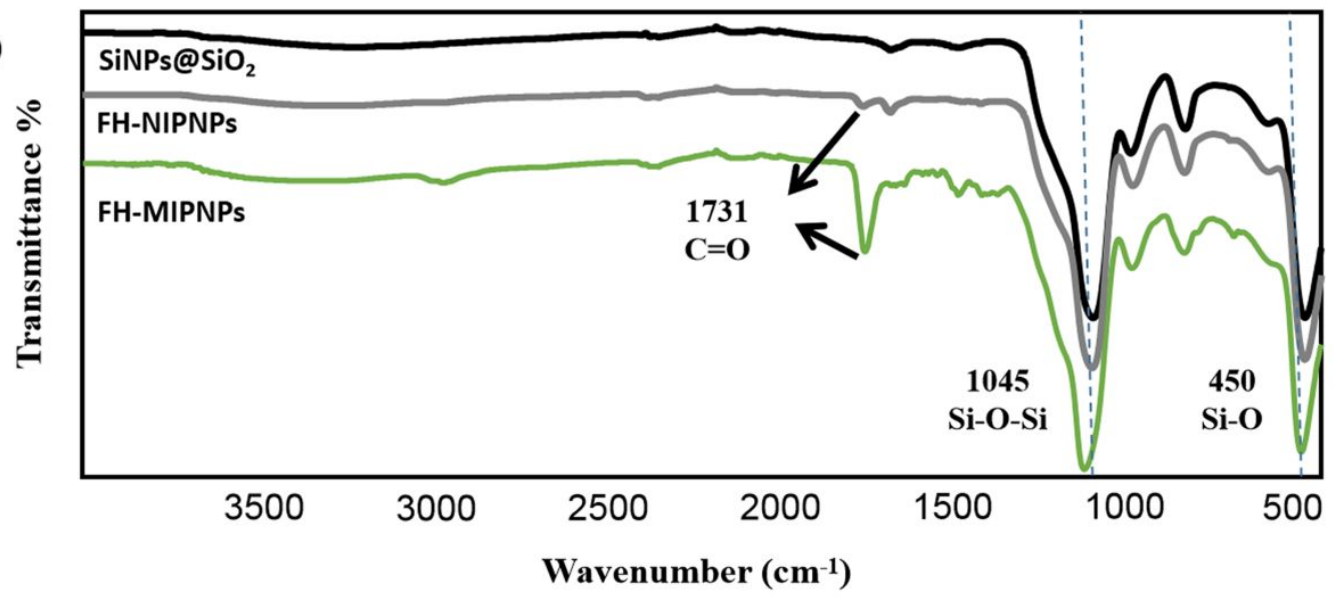

(b)
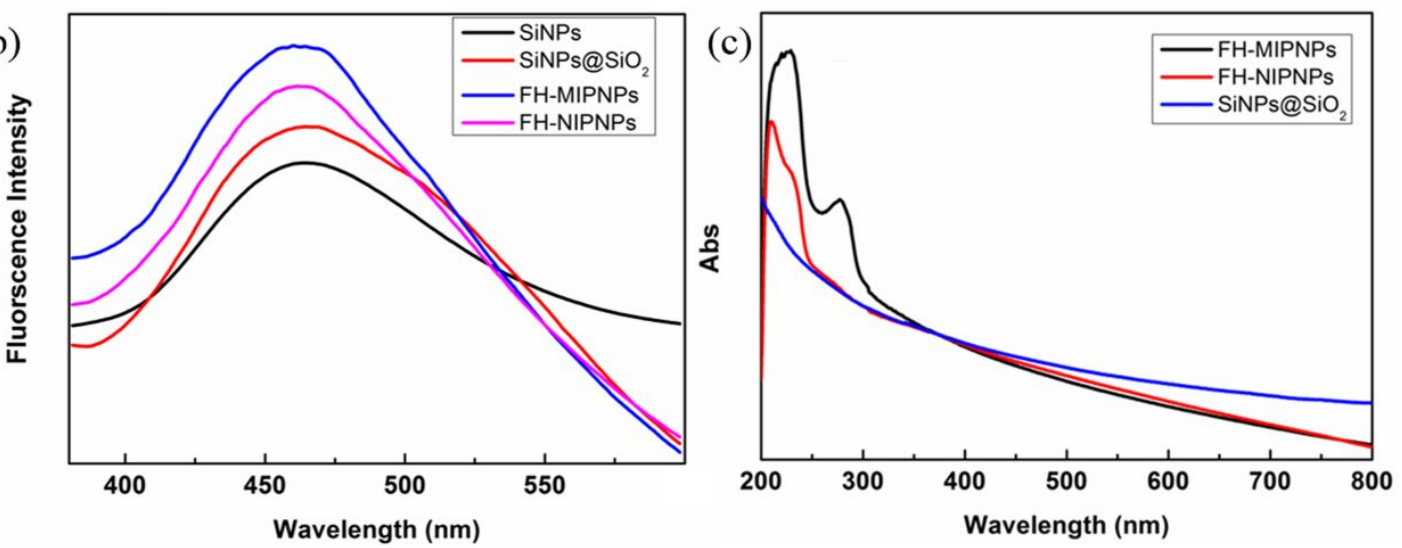

Figure S7. (a) FT-IR spectra of SiNPs@SiO 2 , FH-MIPNPs and FH-NIPNPs; (b) Fluorescence spectra of SiNPs, SiNPs@ $@ \mathrm{SiO}_{2}$, FH-MIPNPs and FH-NIPNPs (Excitation wavelength = $350 \mathrm{~nm}$ ); (c) UV-visible absorption spectra of $\mathrm{SiNPs} @ \mathrm{SiO}_{2}$, FH-MIPNPs and FH-NIPNPs. 


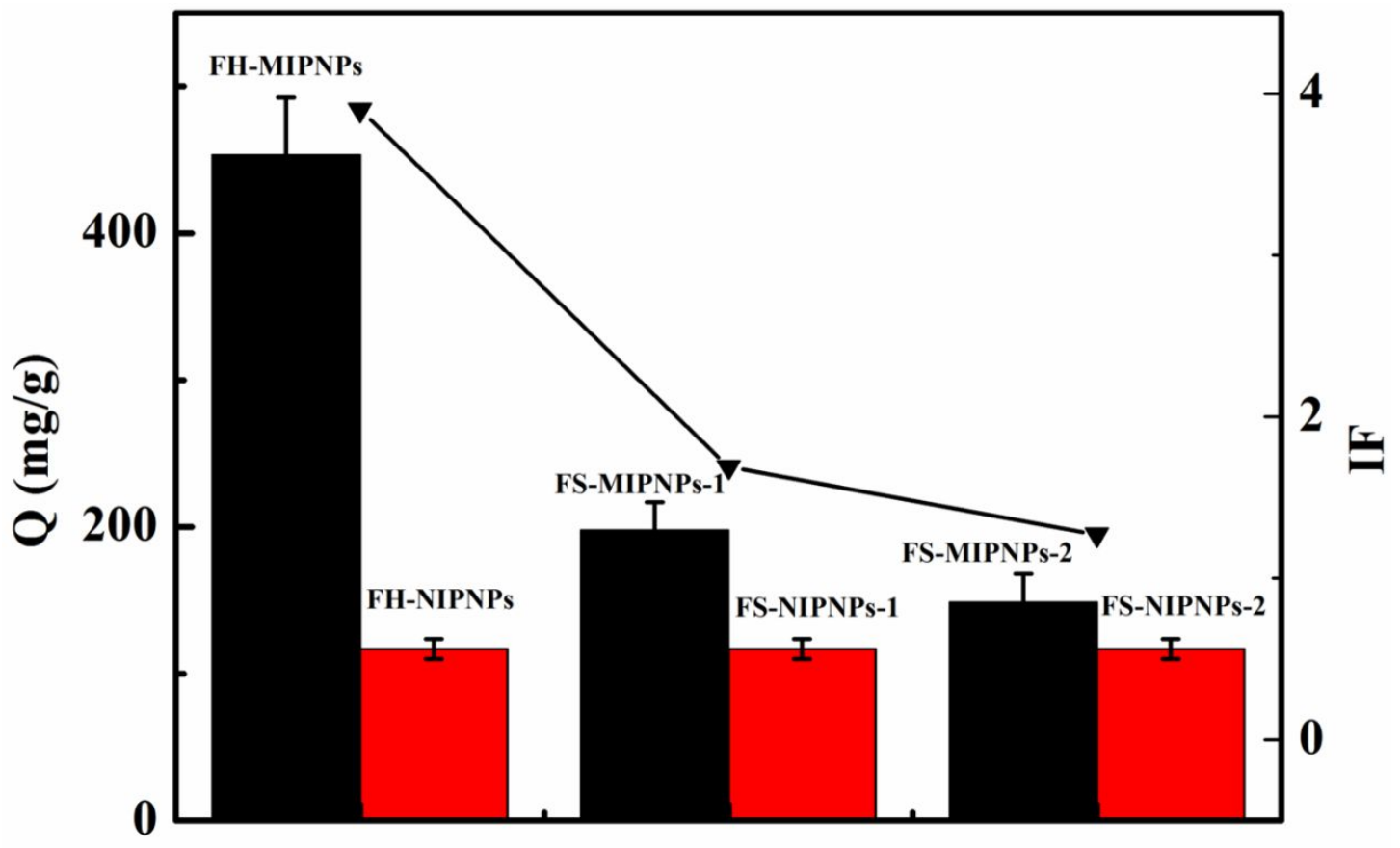

Figure S8. Adsorption of FH-MIPNPs, FS-MIPNPs-1, FS-MIPNPs-2 and FH-NIPNPs on FH. 
Instrument: The ultraviolet-visible absorption spectrum was measured by UV-2450 ultraviolet-visible spectrophotometer (Shimadzu, Japan), and the fluorescence emission spectrum was measured by F-4500 fluorescence spectrophotometer (Hitachi, Japan). The TEM photograph was obtained by HT7700 Exalens microscope (Hitachi, Japan), and the HRTEM image was obtained by JEM-2800 (JEOL, Japan). Scanning electron microscope (SEM) photograph and X-ray energy spectrum analysis (EDS) were measured by JIS-7500F microscope (JEOL, Japan), Fourier transform infrared spectroscopy (FTIR, 4000-400 $\mathrm{cm}^{-1}$ ) was measured by a TENSOR II Fourier Transform Infrared Spectrometer (Bruker, Germany). Cytofluorescence microscopy images were obtained by TCS SP8 laser scanning confocal microscope (Leica, Germany) and thermogravimetric analysis was performed by TGA/DSC1 (Mettler Toledo, Switzerland).

Cell Culture: BxPC-3, Beas-2B and A549 cells were cultured in Dulbecco's modified Eagles medium (DMEM) with 10\% fetal bovine serum and $100 \mathrm{U} / \mathrm{ml} \mathrm{1 \%}$ antibiotics penicillin/streptomycin and maintained at $37{ }^{\circ} \mathrm{C}$ in a $100 \%$ humidified atmosphere containing $5 \% \mathrm{CO}_{2}$.

Western Blot Assay: BxPC-3 cells, A549 cells and Beas-2B cells were cultured in 6-wells plates for $12 \mathrm{~h}$. Cell Lysates were collected after that for Western blot to detect the expression of FN14 on the three kinds of cells.

Establishment of Tumor Model: BxPC-3 cells $\left(5 \times 10^{6}\right)$ were injected subcutaneously to the left inferior temporal region of $\mathrm{BALB} / \mathrm{c}$ nude mice and the tumor model was established. The treatment experiment of the model nude mice was 
started after the tumor grew to a size of $40-100 \mathrm{~mm}^{3}$. Tumor volume was calculated by the formula: tumor volume $=\left[\right.$ length $\left.\times(\text { width })^{2}\right] / 2$. The length and width of each tumor were determined using a vernier caliper. 